\title{
Systematic Review to Compare Urothelium Differentiation with Urethral Epithelium Differentiation in Fetal Development, as a Basis for Tissue Engineering of the Male Urethra
}

\author{
Petra de Graaf, $\mathrm{PhD}^{1,2}$ E. Martine van der Linde, BSc, Peter F.W.M. Rosier, MD, PhD, Ander Izeta, PhD, \\ Karl-Dietrich Sievert, MD, PhD, J.L.H. Ruud Bosch, MD, PhD, and Laetitia M.O. de Kort, MD, PhD ${ }^{1}$
}

\begin{abstract}
Background: Tissue-engineered (TE) urethra is desirable in men with urethral disease (stricture or hypospadias) and shortage of local tissue. Although ideally a TE graft would contain urethral epithelium cells, currently, bladder epithelium (urothelium) is widely used, but morphologically different. Understanding the differences and similarities of urothelium and urethral epithelium could help design a protocol for in vitro generation of urethral epithelium to be used in TE grafts for the urethra.
\end{abstract}

Purpose: To understand the development toward urethral epithelium or urothelium to improve TE of the urethra. Methods: A literature search was done following PRISMA guidelines. Articles describing urethral epithelium and bladder urothelium development in laboratory animals and humans were selected.

Results: Twenty-nine studies on development of urethral epithelium and 29 studies on development of urothelium were included. Both tissue linings derive from endoderm and although adult urothelium and urethral epithelium are characterized by different gene expression profiles, the signaling pathways underlying their development are similar, including Shh, BMP, Wnt, and FGF. The progenitor of the urothelium and the urethral epithelium is the early fetal urogenital sinus (UGS). The urethral plate and the urothelium are both formed from the p63+ cells of the UGS. Keratin 20 and uroplakins are exclusively expressed in urothelium, not in the urethral epithelium. Further research has to be done on unique markers for the urethral epithelium.

Conclusion: This review has summarized the current knowledge about embryonic development of urothelium versus urethral epithelium and especially focuses on the influencing factors that are potentially specific for the eventual morphological differences of both cell linings, to be a basis for developmental or tissue engineering of urethral tissue.

Keywords: urology, urethra, bladder, developmental engineering, systematic review

\section{Introduction}

$\mathbf{U}$ RETHRAL RECONSTRUCTIVE SURGERY is performed in male patients with urethral stricture or congenital disorders such as hypospadias. In hypospadias, the distal part of the urethra is underdeveloped or lacking. In urethral stricture disease, the lumen of the urethra is narrowed due to fibrosis, induced by (iatrogenic) trauma, infection, or inflammation. Surgical correction includes reconstruction using local or more distant autologous tissue, such as prepuce or buccal mucosa. This may result in donor-site morbidity or graft failure. In comparison with distant autologous material, a tissue-engineered (TE) urethral graft might solve the problem of tissue deficit and graft failure. ${ }^{1}$

The urethral wall is composed of three layers: the urethral epithelium, lamina propria, and a layer of smooth muscle cells. The morphology of the layer facing the lumen, the urethral epithelium varies in different parts of the urethra. The membranous and spongy urethra is lined with pseudostratified columnar epithelium lines and near the meatus

\footnotetext{
${ }^{1}$ Department of Urology, University Medical Centre Utrecht, Utrecht, The Netherlands.

${ }^{2}$ Regenerative Medicine Center Utrecht, Utrecht, The Netherlands.

${ }^{3}$ Tissue Engineering Laboratory, Bioengineering Area, Instituto Biodonostia, Hospital Universitario Donostia, San Sebastián, Spain.

${ }^{4}$ Department of Biomedical Engineering, School of Engineering, Tecnun-University of Navarra, San Sebastián, Spain.

${ }^{5}$ Paracelsus Medical University (PMU), Salzburg, Austria.
} 
the epithelium has a more squamous morphology. The prostatic urethra is lined with urothelium, identical to the bladder lumen. The bladder wall presents three layers: urothelium, lamina propria, and smooth muscle. Urothelium is a type of transitional epithelium also lining the ureters and the collecting system of the kidneys. Urothelium in the upper urinary tract is derived from the embryonic mesodermal germ layer, while urothelium in the lower urinary tract (LUT) originates in the endodermal germ layer. ${ }^{2}$

Up until now, urothelium or oral mucosal cells are being used for urethral $\mathrm{TE}^{3}$ while actually the major part of the urethra is lined with urethral epithelium. Currently used TE urethral grafts are not fully successful. ${ }^{3}$ Possibly we lack sufficient understanding of the cellular and molecular differences between the epithelial cells currently used in urethral grafts and urethral epithelium. Developmental engineering, ${ }^{4}$ that is, recapitulation of the developmental cues to generate TE grafts, may improve outcome of TE grafts. The goal of this review is therefore to analyze differences in growth factors and signaling pathways implicated in the development of both urothelium and urethral epithelium, to help design a graft with increased biological and medical impact.

\section{Methods}

\section{Literature search}

A search was done (August 21, 2015) in PubMed/ MEDLINE and EMBASE following PRISMA guidelines. ${ }^{5}$ We used the search terms "(urethral OR urethra) epithelium development" and "urothelium bladder development" restricted to the English language, development and standard laboratory animals or humans. Figure 1 presents an outline of the literature search in a PRISMA Flow Diagram. ${ }^{5}$ For the PRISMA Checklist, ${ }^{5}$ see Supplementary Table S1 (Supplementary Data are available online at www.liebertpub.com/teb).

\section{Study selection}

The results of the search were exported to RefWorks version 2.0 and duplicates were removed. Title screening was done by author PdG. Abstract screening was independently done by PdG and EMvdL. The two screening results were compared and differences were resolved by discussion. Full-text screening was performed by PdG with the assistance of LdK. All studies reporting embryonic and fetal development of epithelial lining of the bladder and the male
FIG. 1. Search strategy and selection of the studies for urothelium $(n=29)$. The database search was performed on August 21, 2015 according to the PRISMA statement [5]. For more details on the search strategy, see the Methods section.
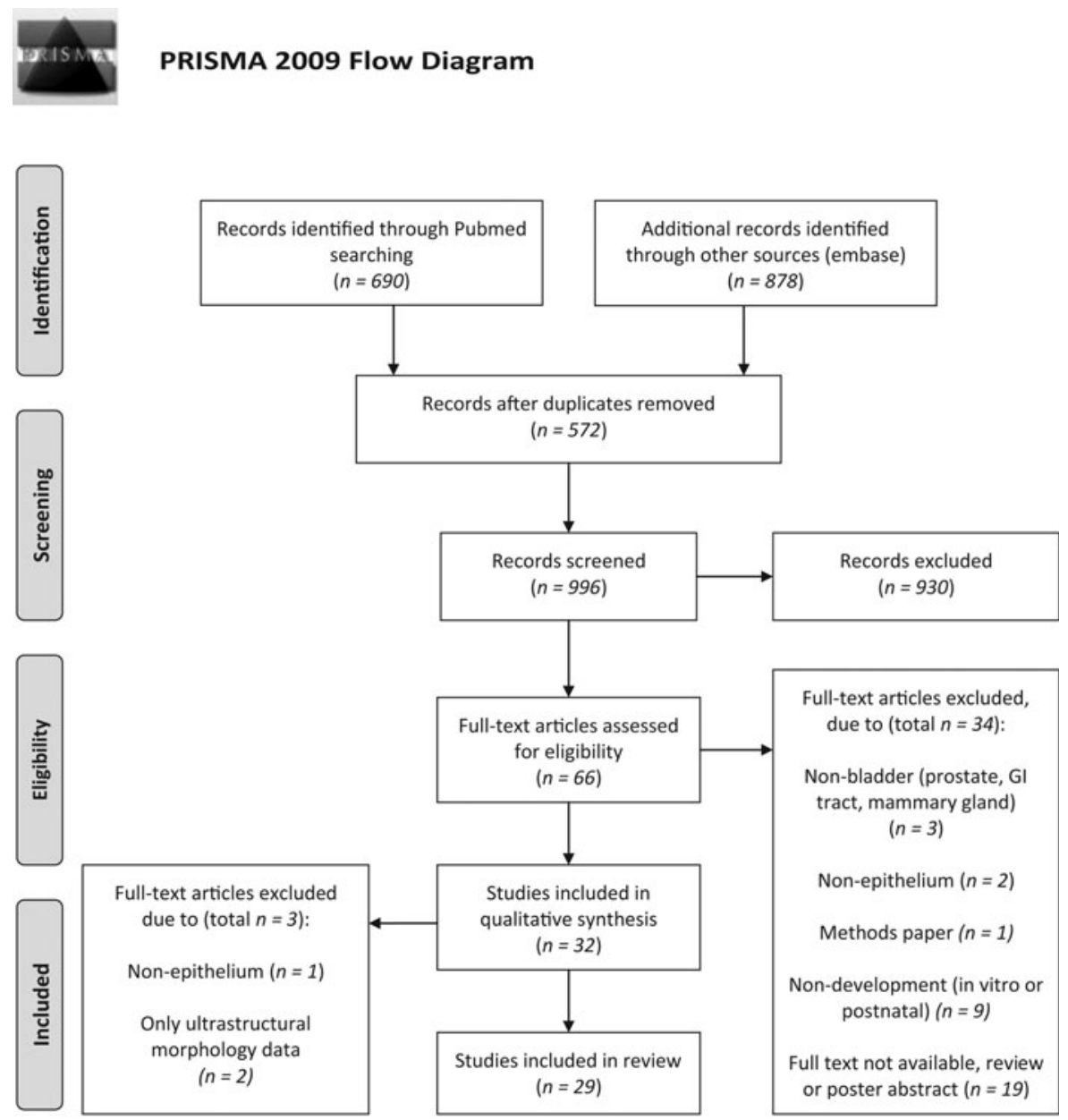

From: Moher D, Liberati A, Tetzlaff J, Altman DG, The PRISMA Group (2009). Preferred Reporting ferms for Systematic Reviews and MetaAnalyses: The PRISMA Statement. PLoS Med 6(6): e1000097. doi:10.1371/journal.pmed1000097 
distal urethra were included. Excluded were articles on carcinogenesis, studies on development of prostate or ureter, studies on adult bladder or urethra, studies on female urethra, and review articles, commentaries, and articles in languages other than English.

\section{Data extraction and analysis}

From the selected studies, the following data were extracted: species, experimental setup, and authors' conclusion. The data were entered in a database using MS office Excel 2010. As the included studies in our review vary in many parameters (e.g., species, experimental setup), no statistical analysis could be performed.

\section{Results}

In total, 996 articles were found on development of urothelium of the bladder, of which 930 were excluded after title screening, mostly due to different determinants (carcinogenesis). Sixty-six articles were subjected to a full-text screen, leading to inclusion of 29 articles in this analysis (24 describing experiments in mice/rats, 3 in humans, and one study combining results from humans and mice) (Fig. 1).
On development of urethral epithelium, only 131 articles were found after removing duplicates. Of these, 52 were subjected to full-text screening, resulting in the inclusion of 29 articles (21 in mice/rats, 8 in humans) in this analysis (Fig. 2). One article was found eligible for both searches. ${ }^{6}$

\section{Embryological development of the bladder and the urothelium}

Table 1 summarizes the results on the development of urothelium.

During early development, the cloaca divides into the urogenital tract and anal canal. The endoderm and mesoderm form most of the lower and upper urinary tract, respectively. During embryogenesis, the cloaca is divided by the urorectal septum, resulting in the urogenital sinus (UGS) on the ventral side of the body and the rectum and anus on the dorsal side. Both the urethra and bladder develop from the UGS derived from the endodermal germ layer. ${ }^{7}$

From the upper part of the UGS, the bladder develops. In a three-dimensional (3D) reconstructive analysis of developing mouse bladders, it was found that at day 13 of embryonic development (E13), bladders were composed of 5\% urothelium, 62\% lamina propria, and 33\% smooth muscle. The percentage of urothelium remained relatively invariable

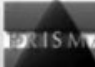

PRISMA 2009 Flow Diagram

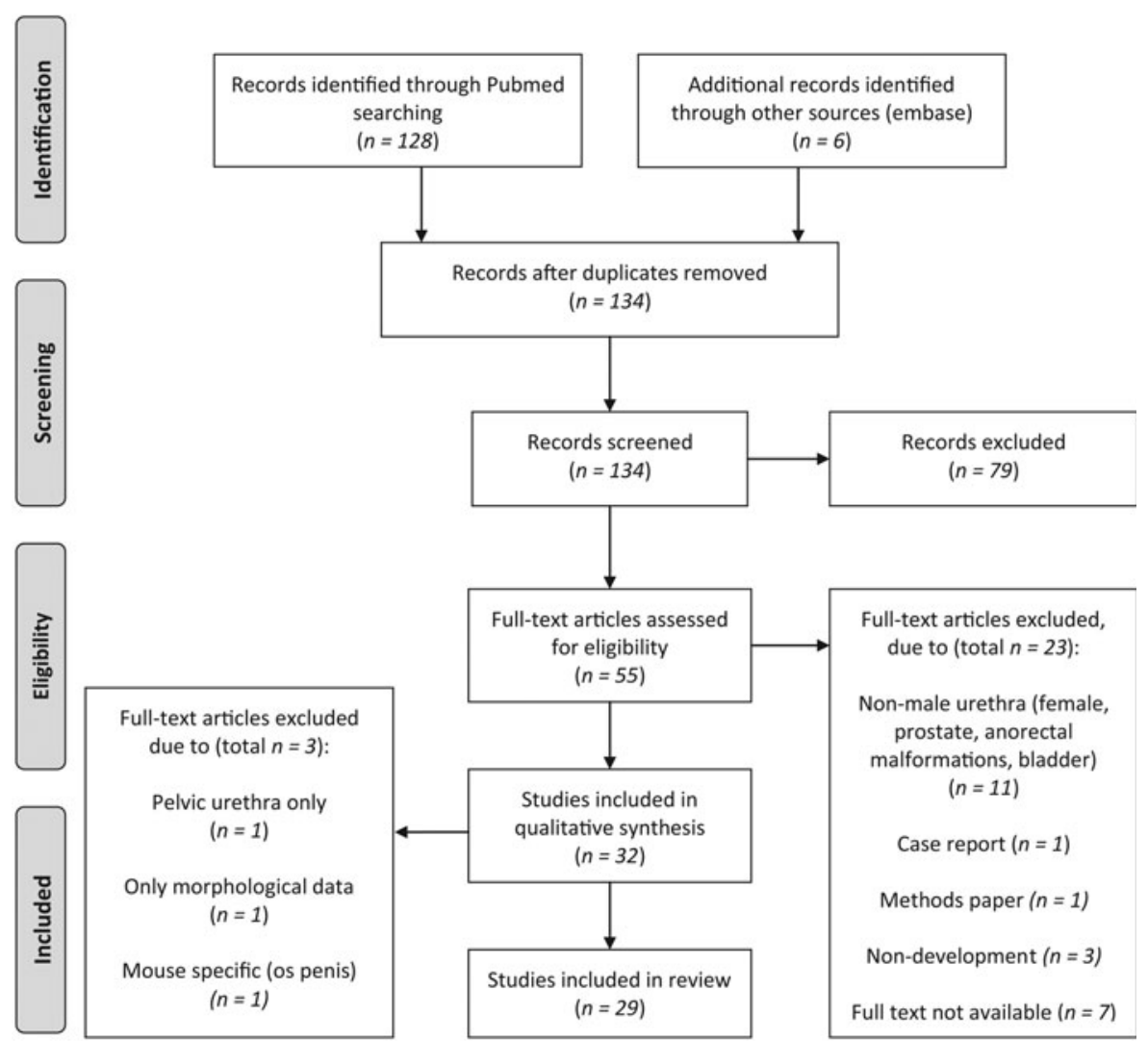

FIG. 2. Search strategy and selection of the studies for urethra epithelium $(n=29)$. The database search was performed on August 21, 2015 according to the PRISMA statement [5]. For more details on the search strategy, see the Methods section. 
Table 1. Results of Systematic Search on Development of Bladder and Urothelium

\begin{tabular}{|c|c|c|}
\hline Authors, year & Species & Type of experiment \\
\hline Abler et al..$^{31}$ & Mouse & $\begin{array}{l}\text { In situ hybridization on an E17 mouse embryo to map LUT gene } \\
\text { expression patterns }\end{array}$ \\
\hline Baskin et al. ${ }^{19}$ & Rat & Tissue recombination experiments \\
\hline Baskin et $a .^{20}$ & Rat & Tissue recombination experiments \\
\hline Bitgood and McMahon ${ }^{6}$ & Mouse & In situ hybridization of the mouse hedgehog gene family \\
\hline Burgu et al. 26 & Mouse & Whole organ culture \\
\hline Burgu et al. ${ }^{27}$ & Mouse & Whole organ culture \\
\hline Cao et al. ${ }^{24}$ & Mouse & Whole organ culture \\
\hline Cao et al. $^{25}$ & Mouse & Whole organ culture \\
\hline Carpenter et al. ${ }^{8}$ & Mouse & $3 \mathrm{D}$ reconstruction of developing mouse bladders \\
\hline Cheng et al. ${ }^{17}$ & Mouse & Analysis of the role of p63 in developing bladder \\
\hline Chung et al. ${ }^{34}$ & Mouse & miRNA expression profiles in urinary tissues \\
\hline Colopy et al. ${ }^{12}$ & Mouse & BrdU labeling to identify the label-retaining cells (LRC) \\
\hline De La Rosette et al. ${ }^{9}$ & Human & Investigation of the keratin phenotype of the human bladder epithelium \\
\hline DiSandro et al. ${ }^{28}$ & Mouse & Role of EGF in urothelial development \\
\hline DiSandro et al. ${ }^{21}$ & Rat & Tissue recombination experiments \\
\hline Erman et al. ${ }^{10}$ & Mouse & Immunohistochemistry of developing mouse bladders \\
\hline Gandhi et al. ${ }^{13}$ & Mouse & $\begin{array}{l}\text { Gene expression, tracing, and analysis of label-retaining cells } \\
\text { in mouse embryos }\end{array}$ \\
\hline Georgas et $a l_{.}^{32}$ & Mouse & Immunohistochemistry and gene tracing in mouse embryos \\
\hline Jenkins et al. $^{23}$ & Human & Immunohistochemistry of Shh and downstream targets of Shh \\
\hline Karni-Schmidt et al. ${ }^{16}$ & $\begin{array}{l}\text { Mouse } \\
\text { and human }\end{array}$ & Analysis of p63 expression using isoform-specific antibodies \\
\hline Pignon et al. ${ }^{15}$ & Mouse & Gene expression tracing and reporter assays \\
\hline Rudat et al. ${ }^{11}$ & Mouse & Gene expression tracing and reporter assays \\
\hline Shiroyanagi et al. ${ }^{22}$ & Mouse & $\begin{array}{l}\text { Gene expression and protein expression in ex vivo embryonic } \\
\text { bladder cultures }\end{array}$ \\
\hline Sun et al. ${ }^{14}$ & Mouse & EdU/BrdU labeling to identify the label-retaining cells (LRC) \\
\hline Tanaka et al. ${ }^{7}$ & Mouse/rat & Tissue recombination experiments \\
\hline Thomas et al. $^{33}$ & Rat & Tissue recombination experiments \\
\hline Vinter-Jensen and Nielsen ${ }^{29}$ & Rat & Role of EGF in urothelial development \\
\hline Vinter-Jensen and Orntoft ${ }^{30}$ & Rat & Role of EGF in urothelial development \\
\hline
\end{tabular}

throughout development, while the percentage of lamina propria decreased to $28 \%$ mirrored by a corresponding increase in the percentage of bladder smooth muscle to $67 \% .^{8}$ The urothelium consists of multiple cell layers in different stages of differentiation. At the luminal side of the bladder, a layer of "umbrella cells" can be found. Deeper layers are formed by intermediate and basal cells. The latter cells are cubical and round cells, while the umbrella cells are very large and binucleated.

Expression of keratins and uroplakins. In a study with human embryonic bladders, it was shown that expression of keratins in the urothelium changes with gestational age, ${ }^{9}$ consisting of focal loss of keratin 7, restriction of keratin 20 expression to umbrella cells, and expression of keratin 18 throughout the full thickness of the urothelium. Keratin 5 was expressed in the basal cell layer, while keratins 14 and 17 were not found. Squamous cell keratin 13 was found throughout the full thickness of the urothelium. ${ }^{9}$ In addition to determination of gestational age, keratins are also useful as differentiation markers. Superficial cells expressing keratin 7 and 20 will appear on E18 marking the termination stage of mouse embryonic differentiation, indicating that apical membrane specialization is a differentiation marker for cells. ${ }^{10}$ In addition, these superficial umbrella cells are positive for uroplakins. Uroplakin 3B (Upk3b) is specifically expressed both in development and under homeostatic con- ditions in adult mice in the mesothelia of the body cavities, that is, the epicardium and pericardium, the pleura and the peritoneum, and in the urothelium of the urinary tract. ${ }^{11}$ To analyze Upk3b function, $U p k 3 b$ expression was genetically abrogated. Analyses of these $U p k 3 b$-deficient mice did not reveal changes in differentiation or integrity of the urothelium and the mesothelia that cover internal organs. As $U p k 3 b$ is coexpressed with the closely related $U p k 3 a$ gene in the urothelium, redundancy could explain the lack of effects. ${ }^{11}$

Three studies combined identification of label retaining cells with marker analysis. All identified the basal cells as the progenitor cell type. ${ }^{12-14}$ The cells in the basal layer of the urothelium are able to proliferate and subsequently differentiate into intermediate and luminal (or umbrella) cells, thereby regenerating bladder tissue. These basal cells are stem cell like; they proliferate and differentiate into either intermediate or umbrella cells. A prominent feature of basal cells is the expression of keratin $5 .{ }^{15}$ Taken together, keratins and uroplakins can be used to distinguish the different cell types in the bladder. The umbrella cells express uroplakin and keratin 20 . The basal cells of urothelium are characterized by keratin 5 and 14 expression, while intermediate and luminal cells express keratin 7,14 , and $20{ }^{12,14}$

Role of p63 in bladder and urothelial development. The p63 protein is a transcription factor inducing stem cell properties in epithelial cells. By alternative promoter use, 
two main p63 isoforms are generated with different transcription activities, including the TA isoforms, in which the $\mathrm{N}$-terminal transactivation domain is retained, and the $\Delta \mathrm{N}$ isoforms, which lack the TA domain. Analysis of murine fetal and newborn bladders with isoform-specific antibodies revealed that the first and most expressed isoform is TAp63, appearing at E16.5. Only $24 \mathrm{~h}$ after birth, $\Delta \mathrm{Np} 63$ expression is detected in the basal and intermediate layers of mouse urothelium, indicating that balancing p63 isoform expression is important during urothelial development. The umbrella cells in the superficial, luminal layer display a p63negative phenotype. ${ }^{16}$

In a lineage tracing study using an injury model, the cell type responsible for regeneration was identified; the intermediate cells gave rise to the new urothelial cells, instead of the basal cells. ${ }^{13}$ Although seemingly contradictory at first, these findings are not surprising. Intermediate cells are more differentiated than basal layer cells, but still have some stem cell-like characteristics such as $\operatorname{Sh}$ (see next section "Sonic hedgehog") and p63 expression. Gandhi and colleagues concluded that p63 expression (although no isoform differentiation was made) is the key marker for stemness in these cells. ${ }^{13}$

While the group of Karni-Schmidt et al. ${ }^{16}$ could only detect $\Delta$ Np63 after birth using antibodies, in a Cre-lox recombination technique in mice to express $\Delta$ Np63 cell lineages in knockout mice was revealed that $\Delta \mathrm{Np} 63$ is already present in the UGS. Urothelium in the bladder and prostate was shown to derive from the $\Delta \mathrm{Np} 63+$ cells in the UGS, ${ }^{15}$ denoting these cells as progenitor cells, or P-cells. These cells are present during bladder development until (mouse) E18 and give rise to umbrella and intermediate cells of urothelium. ${ }^{15}$ P-cells are p63+, but also express uroplakins. It was proposed that intermediate cells and basal cells can both function as progenitors, ${ }^{15}$ which is in line with the study of Gandhi. ${ }^{13} \Delta \mathrm{Np} 63+$ cells are also present in rectal epithelium, suggesting that the $\Delta \mathrm{Np} 63+$ cell originates in the fetal cloaca. $^{15}$

p63-null mice develop an abnormal urothelium composed of a single cell layer that, based on the keratin expression pattern, consists of luminal cells with umbrella phenotype. ${ }^{16}$ Cheng et al. found $\triangle N p 63$ to be the predominant form of p63 in the developing bladder. In p63 knockout mice, increased apoptosis of urothelium was found in combination with the absence of abdominal and ventral bladder walls. ${ }^{17}$ These defects resemble bladder exstrophy, a congenital anomaly exhibited in human neonates, as confirmed by genetic analysis of a cohort of bladder exstrophy epispadias complex patients. ${ }^{18}$

Sonic hedgehog. The urothelium is surrounded by the lamina propria and the smooth muscle layers of the musculus detrusor. In a grafting experiment using chimeric bladders, it was shown that cell-cell interactions between the bladder mesenchyme and the urothelium are necessary for the induction of smooth muscle and normal bladder development. ${ }^{19,20}$ Mature urothelium and heterotypic epithelium also induce smooth muscle differentiation. ${ }^{21}$

Sonic hedgehog (Shh) is one of the factors important for the smooth muscle layer differentiation in mice ${ }^{22}$ and in humans. ${ }^{23}$ Shh is expressed in different types of epithelial cells that interact with mesenchymal cells. ${ }^{6}$ Mechanistically, Shh acts as an autocrine growth factor and promotes mes- enchymal proliferation. Then, once a critical mass of mesenchymal cells has been achieved, Shh acts through the Gli proteins and BMP4 to induce smooth muscle differentiation. ${ }^{24,25}$ Abnormal development of the detrusor muscle is observed in the absence of urothelium, underlining the relevance of Shh signaling during bladder development. ${ }^{24,25}$

Growth factors. An important growth factor for the differentiation of UGS endoderm into urothelium is the vascular endothelial growth factor (VEGF). ${ }^{26}$ VEGF and its receptors are mainly expressed around E14, at which stage the urothelium is still developing. VEGFR1 is known for its involvement in angiogenesis and is expressed predominantly in epithelial cell lineages. Immunohistochemical staining revealed that VEGF contributes to urothelial differentiation by inhibiting apoptosis and promoting proliferation. ${ }^{26}$ The relevance of VEGF was later confirmed by organ cultures showing that the addition of VEGF resulted in extra growth of the whole bladder in vitro. ${ }^{27}$

On the contrary, the epidermal growth factor (EGF) was not essential for urothelial regeneration after injury. ${ }^{28}$ However, EGF induces increased volume of all wall layers, most prominently the urothelium, maintaining the normal pattern of differentiation. ${ }^{29,30}$

Other factors involved. Two studies integrated the data from current literature to make an overview of the genitourinary tract. First, Abler et al. used in situ hybridization for mapping LUT gene expression patterns. ${ }^{31}$ Subcompartments in the urothelium include the following layers: basal (Kremen1+; Krt14+), intermediate (Kremen1+; Upk1b+), and superficial urothelium (Kremen1-; Upklb+). Second, Georgas et al. used data from the GUDMAP project in combination with 3D imaging techniques to complete the anatomical ontology of the LUT. ${ }^{32}$ The urothelium expresses a series of endodermal markers (Shh, Trp63, Isl1, and Foxa2). From E14, the ontology subdivides urothelium into superficial, intermediate, and basal cell layers. Krt5+ cells are first identified in intermediate and basal layers between E14 and E15 and are occupying the entire basal layer by E17.

In a xenograft approach, Thomas et al. removed urothelium from the underlying mesenchyme from embryonic day 18 rat bladders and generated heterospecific recombinant xenografts by combining mouse embryonic stem cells with rat embryonic bladder mesenchyme. After 16 days, uroplakin was detectable and correlated with the loss of Foxa2, while Foxa1 remained throughout development. Androgen receptor (AR) was first noted in stroma at day 16 . It localized to urothelial nuclei at day 21 and was undetectable at 42 days. Adjacent to the urothelium, $\alpha$-smooth muscle actin was seen on day 16 and it was localized in bundles to the periphery of the graft at later time points.

Staining urothelium confirmed basal orientation of p63 throughout differentiation of the urothelium. ${ }^{33}$ To improve our understanding of urothelial differentiation, Chung et al. analyzed the microRNA (miRNA) expression profiles of mouse urinary tissues. In the undifferentiated basal and intermediate cell layers, miR-205 was enriched. In a proteomic approach, 24 genes were identified to be regulated by miR-205; eleven of them were annotated as cell junction- and tight junction-related molecules. Knockdown of miR-205 in urothelial cells showed alterations in keratin synthesis and 
Table 2. Results of Systematic Search on Development of Urethra and Urethra Epithelium

\begin{tabular}{|c|c|c|}
\hline Authors, year & Species & Type of experiment \\
\hline Beleza-Meireles et al. ${ }^{57}$ & Human & Genetic mutation analysis in patient cohort \\
\hline Bitgood and McMahon ${ }^{6}$ & Mouse & In situ hybridization of the mouse hedgehog gene family in embryos E11.5-16.5 dpc \\
\hline Chen et al. ${ }^{50}$ & Mouse & $\begin{array}{l}\text { Organ explant culture, in situ hybridization, and morphology study in normal and } \\
\text { Fkbp52-deficient mice }\end{array}$ \\
\hline Ching et al. ${ }^{55}$ & Mouse & Analysis of expression of Spry 1 and Spry 2 in mouse embryos and deletion of the genes \\
\hline Chiu et al. ${ }^{37}$ & Mouse & $\begin{array}{l}\text { Gene expression in the LUT, microarray data in combination with whole mount in situ } \\
\text { hybridization in mice at E13 and E14 dpc }\end{array}$ \\
\hline Erol et al. ${ }^{62}$ & Human & Immunohistochemical analysis of the fetal human penis. \\
\hline Gredler et al. ${ }^{58}$ & Mouse & Study of the external genital development by conditional deletion of Fgfr2 \\
\hline Hadidi et al. $^{40}$ & Human & Immunohistochemical analysis of the fetal human penis \\
\hline Harada et al. $^{59}$ & Mouse & $\begin{array}{l}\text { In situ hybridization, histology, and immunohistochemistry in mice carrying deletions } \\
\text { in the FGF pathway }\end{array}$ \\
\hline Haraguchi et al. ${ }^{54}$ & Mouse & $\begin{array}{l}\text { In situ hybridization of components of the FGF system, genital tubercle organ culture, } \\
\text { Fgf10 deletion }\end{array}$ \\
\hline Hynes and Fraher ${ }^{36}$ & Mouse & Morphological study in mouse embryos using EM and light microscopy \\
\hline Hynes and Fraher ${ }^{39}$ & Mouse & Morphological study in mouse embryos using EM and light microscopy \\
\hline Jesmin et al. ${ }^{52}$ & Rat & Analysis of expression levels of ER $\alpha$ and ER $\beta$ in the penis \\
\hline Kim et al. ${ }^{51}$ & Human & Immunohistochemical analysis of the fetal human penis \\
\hline Kurzrock et al. ${ }^{35}$ & Human & Immunohistochemical analysis of the fetal human penis \\
\hline Li et al. & Mouse & Gene expression profiles of the genital tubercle $(\mathrm{GT})$ at E15, E16, and E17 dpc \\
\hline Li et al. ${ }^{41}$ & Human & Immunohistochemical analysis of the fetal human penis \\
\hline Miyagawa et al. $^{48}$ & Mouse & $\begin{array}{l}\text { In situ hybridization of components of the Shh pathway in the GT both in normal } \\
\text { and Gli2- or Gli3-deficient mice }\end{array}$ \\
\hline Morgan et al. ${ }^{47}$ & Mouse & In situ hybridization of components of the Shh pathway in Hoxa13-deficient mice \\
\hline Ogino et al. $^{42}$ & Mouse & In situ hybridization of genes in the FGF and RA signaling systems \\
\hline Pechriggl et al. ${ }^{61}$ & Human & Immunohistochemical analysis of the fetal human penis \\
\hline Perriton et al. ${ }^{45}$ & Mouse & In situ hybridization, tissue grafting, and scanning electron microscopy \\
\hline Petiot et al. & Mouse & Knockout model (Fgfr2-IIIb-/-) \\
\hline Sajjad et al. ${ }^{49}$ & Human & Immunohistochemical analysis of androgen receptor expression in the LUT \\
\hline Satoh et al. ${ }^{53}$ & Mouse & Analysis of expression of FGF genes and FGF receptor genes in the GT of mice \\
\hline Seifert et al. ${ }^{43}$ & Mouse & Fate mapping of the penile urethra by following Shh expression \\
\hline Seifert et $a l^{44}$ & Mouse & Conditional knockout of FGF8 \\
\hline $\mathrm{Wu}$ et $a l .{ }^{46}$ & Mouse & Gene tracing in a BMP7 mouse strain \\
\hline
\end{tabular}

increases of uroplakin Ia and Ib, which are the urothelial differentiation products, suggesting that miR-205 contributes to the regulation of urothelial differentiation. ${ }^{34}$

\section{Embryological development of the urethra and the urethral epithelium}

Table 2 summarizes the results on the development of the urethra and the urethral epithelium.

Formation of the proximal and distal male urethra is traditionally regarded as two separate processes. As the epithelium of the distal urethra has a squamous characterization, it was generally accepted that the most distal part of the urethra was that of ectodermal ingrowth. However, at the turn of the century, Kurzrock et al. showed in human embryos that the entire male urethra is of endodermal origin. ${ }^{35}$ They also demonstrated that urethral epithelium from endodermal origin could be induced to differentiate into a squamous phenotype in xenografts of rats and mice. ${ }^{35}$ In fact, the male prostate, prostatic urethra, and membranous urethra develop from the pelvic UGS. The phallic UGS develops into the male urethral plate. ${ }^{36}$

This is verified by a microarray expression study on the different parts of the urogenital system, where the UGS and urethral tissue cluster close together. ${ }^{37}$ Urethral plate epithelium can be found in E10.5 $5^{38}$ and extends in a distal direction to the tip of the glans. ${ }^{39}$ Up until E15, the genital tubercle, the embryonic precursor of the penis and the clitoris, is indistinguishable between males and females. The urethral plate becomes a tube when urethral folds flanking the plate fuse, creating a lumen in-between at late E16 and extending to the glans penis until E18, when the lumen of the urethral plate opens at the glans and the early urethra is formed. ${ }^{39}$ These folds consist of the endoderm and surrounding mesoderm. Hypospadias may arise due to incomplete closing of these urethral folds. ${ }^{36}$

In two separate studies, it was shown that urethral development is evolutionary conserved from mice to humans. During urethral formation, the solid epithelial plate forms a groove, the urethral groove, which is opened distally through canalization, followed by a groove closure to form the tubular urethra. In human embryo studies, no indication of ectodermal intrusion of epidermal cells that meet the solid or canalized urethral plate or urethral groove has been found. ${ }^{40,41}$

Sonic hedgehog. Further evidence for the endodermal origin of the urethral epithelium is given by $S h h$ tracing in mouse models. It is expressed that in the UGS epithelium and in the adjacent mesenchyme the downstream effector BMP4 is expressed. Shh and BMP4 are present in the whole LUT. ${ }^{6,42}$ By tracing Shh expression, it was found that cloacal endoderm gives rise to the whole urethra. ${ }^{43}$ Furthermore, Shh mutations give rise to malformations in the urinary tract in 
mouse models. ${ }^{44}$ The epithelium of the urethral plate expressing Shh is thought to act as an organizing center in urethral development. ${ }^{45}$ Transplantation of this region induced the growth of folding structures similar to that in the developing urethra. ${ }^{37}$ It was proposed that $F G F 8$ and $B M P 7$ are regulated by Shh signaling because these genes exhibit the same expression pattern as $S h h^{37,46}$ Both $F G F 8$ and $B M P 7$ are probably downstream of Shh in the signaling cascade, and deficient mice presented with hypospadias. ${ }^{47}$

Mesoderm is indirectly involved in urethral development. Invasion of stromal mesoderm (connective tissue) into the urethral plate enables masculinization of the developing male urethral plate. Signals from the adjacent and invading mesoderm cause septation of the urethral plate by inducing cell reorganization. ${ }^{43}$ The Shh-Gli2 pathway is responsible for this masculinization process. ${ }^{48}$ The Gli2 gene is expressed in mesenchyme around the urethral epithelium, underlining the importance of epithelial-mesenchymal interaction, especially the expression of Shh and multiple downstream targets, for normal urogenital and urethral development.

Nuclear hormone receptors and their ligands. The AR is a key factor in the development of male genital, but no differences are observed in the distribution of AR between human male and female embryos up to 12 weeks of gestation. ${ }^{49}$ AR initially appears in the mesenchyme during morphogenesis of the genital structures, followed by the appearance of these receptors in the epithelium after around 9 weeks of gestation, concomitant with its functional differentiation. ${ }^{49}$ From E13.5 onward, androgens are crucial for masculinization of the mouse embryo. ${ }^{50}$ The ARs were most expressed on the ventral side of the developing urethra and around the time the urethral folds close,${ }^{51}$ indicating that their expression is required for this closure and development of the glans and scrotum.

Anomalies in androgen signaling are associated with hypospadias in mice. ${ }^{50}$ Mutations in androgen pathway result in feminization of male genitalia (hypospadias or micropenis). Noticeably, in a study in rats, both estrogen receptor alpha and estrogen receptor beta (ER $\alpha$ and ER $\beta)$ are age dependently expressed in the rat penis. ${ }^{52}$ Initial signals (fetal d17) of $\mathrm{ER} \alpha$ were localized in the mesenchyme and subepithelial stroma and later (postnatal d2) in the corpus spongiosum, corpus cavernosum, and urethral epithelium. ER $\beta$ was initially detected by postnatal $\mathrm{d} 2$ and was localized diffusely in corpus spongiosum and cavernosum in immature rats. In adult rats, both ERs were concentrated largely in the urethral epithelium and vascular and neuronal structures.

Fibroblast growth factor. Fibroblast growth factor (FGF) signaling is involved in the development of external genitalia, as it is in limb development. For instance, FGF10 defective mice exhibit hypospadias, ${ }^{38,53}$ confirming that FGF10 is essential for urethral development. ${ }^{54}$ Not only FGF but also modulators of FGF signaling were involved in the development of the urethra. This was shown by deletion of the genes encoding Sprouty (Spry 1 and 2) in mice. ${ }^{55}$ Sprouty proteins are inhibitors of FGF signaling. In normal circumstances, these two genes are expressed in urethral epithelium during embryogenesis and contribute to formation of the urethral lumen by correct fusion of the urethral folds. Spry defective mice developed no urethra at all, but exhibited increased epithelial proliferation, creating abnormal organization within the phallus. ${ }^{55}$

Urethral plate epithelium was characterized by expression of FGF receptor 2 (FGFr2). FGFr2-negative mice had severe hypospadias, indicating the necessity of $F G F r 2$ in the developing urethra. ${ }^{38}$ The loss of AR signaling and FGF10 expression is associated with the same phenotype, showing that FGFr2 and FGF10 are downstream of AR in the molecular cascade in urogenital development. ${ }^{38}$

The loss of $F G F 8$ did not affect urethral development in a conditional knockout mouse model. ${ }^{56}$ On the contrary, in a genetic analysis of a human cohort of hypospadias patients, variations in the $F G F r 2$ and in the $F G F 8$ genes were found to be more prevalent in the hypospadias than in healthy controls. ${ }^{57}$ In the developing genitalia, the expression of different FGF proteins differs between the endoderm, mesoderm, and ectoderm. Although the urethra is entirely of endodermal origin, ${ }^{35,40,41}$ conditional deletion of $F G F r 2$ in mice results in two distinct phenotypes: ectodermal deletion of $F G F r 2$ results in the most severe hypospadias and absence of the ventral prepuce, whereas endodermal $F G F r 2$ deletion causes mild hypospadias and inhibits maturation of a complex urethral epithelium.

This shows a major role for FGFr2 in the developing genital surface epithelium, where maturation of ectodermalderived epithelium is required for maintenance of a closed urethral tube. ${ }^{58}$ This topical distribution is temporarily regulated: first around E11.5, mesenchymal FGF signaling is required for GT outgrowth, then at E13.5, the ectodermal FGF signaling is necessary for urethral tube formation followed by endodermal FGF signaling, which is required for the stratification and adhesive characteristics of urethral epithelium after E14.5. ${ }^{59}$

Other factors involved. Based on expression profiles, transforming growth factor (TGF) and Wnt-Frizzled signaling may be involved in urethral tube formation. ${ }^{60}$ Frizzled 1 and TGF $\beta 1$ are expressed in the adult urethral epithelium, indicating those as potential urethral markers. During early embryonic development, proteins from the urethral plate interact with proteins on the adjacent mesenchymal cells, activating signaling cascades that are very complex and therefore hard to map. By comparing spatial expression patterns in mice, a network of Wnt7a-associated GT-enriched epithelial genes were predicted, including (among others) Krt5 (the gene encoding keratin 5). ${ }^{37}$ As several of the Wnt7a-associated genes are regulated by retinoic acid, estrogen, or androgen, this pathway may be involved in the later androgen-dependent development of the GT. The link to androgen regulation suggests that at least some forms of hypospadias or other congenital glans malformations involve disruptions of epidermal development, ${ }^{37}$ based on the ectodermal deletion of FGFr $2 .{ }^{58}$

In human fetuses, p63 was expressed in the urethral epithelium throughout the entire period of development, with restricted expression in the basal cells from the beginning of W11. ${ }^{61}$ By then, the UGS had differentiated into the bladder and the urethra. Urethral epithelium was characterized by the expression of keratin 7. This protein is also expressed in intermediate cells of the urothelium (see section "Expression of keratins and uroplakins"). The umbrella cells that line the lumen of the bladder and their characteristic uroplakin and 
cytokeratin 20 expression are absent in the urethra. ${ }^{61}$ A study comparing normal human fetal penises with hypospadias revealed differences between the normal urethral epithelium and hypospadias urethral plate epithelium: both keratin 7 and 18 (markers for endodermal origin) were present in normal urethra and absent in the urethral plate of hypospadias. The epithelium of the urethral plate in these hypospadias fetuses resembled more penile skin and preputium, being positive for keratin 14 , a marker for ectodermal origin. ${ }^{62}$

\section{Discussion}

This review summarizes literature on the development of both urothelium and urethral epithelium. Both epithelium types are morphological distinct but do derive from the endodermal layer. Although adult urothelium and urethral epithelium are characterized by different expression profiles, the signaling pathways underlying their development are similar, especially with regard to Shh, BMP, Wnt, and FGF. The last common progenitor cell lineage of the urothelium and the urethral epithelium is the p63+ cells of the UGS, from which the urethral plate and urothelium are formed. Later, during differentiation, basal cells in both urothelium and urethral epithelium cells remain p63+, but the role of the specific isoforms (TAp63 vs. $\Delta \mathrm{Np} 63$ ) remains uncertain.

Keratin 5, 7, and 18 expression is shared between the bladder and urethra, but keratin 20 is never present in the urethral epithelium; this marker is restricted to the umbrella cells in the urothelium, which also express the uroplakins. Unique urethral epithelial markers may be Frizzled1 and components of the TGF $\beta$ pathway, ${ }^{60}$ but these markers were not tested in urothelium. Recently, 12 unique upregulated genes were identified in the urethral plate of mice at E12.5, ${ }^{63}$ but expression data of these genes later in development in urethral epithelium are not identified yet. To fully characterize and distinguish the two types of epithelium, a panel of markers should be developed based on unique expression signatures.

During embryonic development, cell fate depends on intrinsic programs in combination with signals from the cell niche. As a consequence, stem cells can be directed to a specific cell type or tissue by either exposure to a conditioned medium from differentiated cells or by coculturing the stem cells together with differentiated cells. However, these processes are difficult to control in vitro at present and not translatable to regenerative medicine applications. ${ }^{64}$

Better controlled and chemically defined is the developmental engineering technique-directed differentiation (reviewed in Ref. ${ }^{65}$ ). Pluripotent stem cells (PSCs) are cultured in controlled conditions involving specific substrate or extracellular matrices promoting cell adhesion and differentiation, and define culture media compositions. A limited number of signaling factors, such as growth factors or small molecules, controlling cell differentiation are applied sequentially or in a combinatorial manner, at varying dosage and exposure time. Detailed knowledge of the transcription factor expression profiles involved will facilitate the control of cell identity. ${ }^{66}$

Network biology is emerging, and large data sets are currently combined in platforms such as CellNet ${ }^{67}$ and Mogrify. ${ }^{68}$ Although data sets for urogenital tissue are still limited, few protocols have enrolled recently for directed differentiation toward urothelium. ${ }^{69-71}$ These three protocols have in common that the PSCs arrive at an intermediate state, the definitive en-

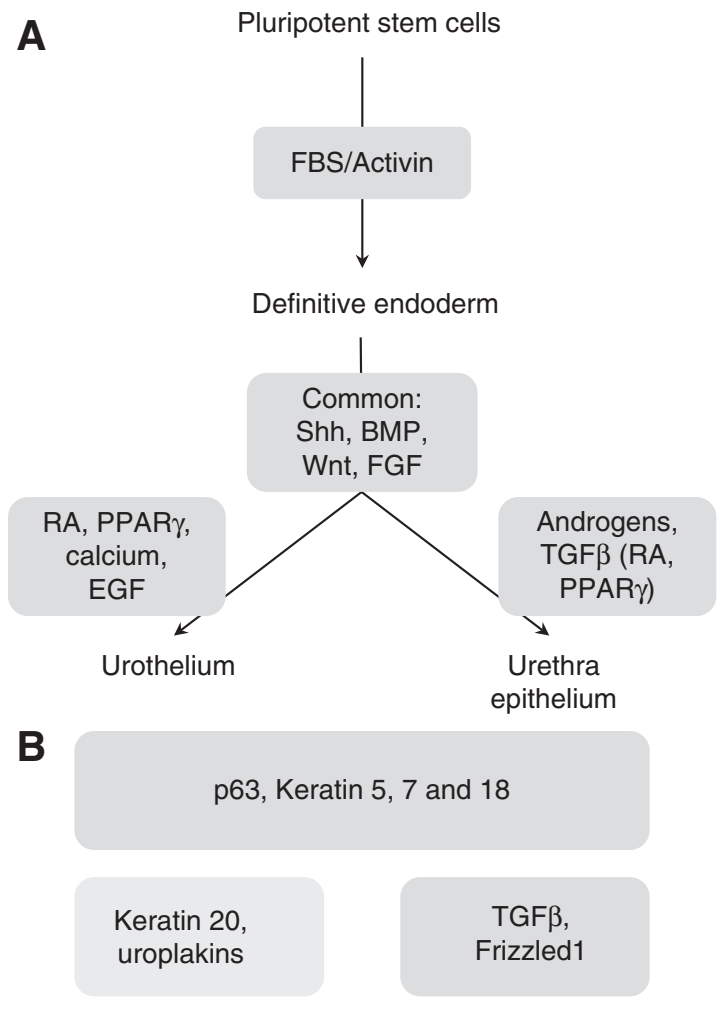

FIG. 3. (A) Suggested protocol for directed differentiation of pluripotent cells toward urothelium and urethra epithelium, based on Osborn et al. ${ }^{70}$ and results from the literature search described in this review (B). Shared (upper panel) and unique (lower panel) markers for urothelium and urethra epithelium. For more details, see the Discussion section.

doderm, before differentiating toward urothelial cells. Based on these studies and the results described in this review, a protocol can be designed for directing PSCs toward urethral epithelium (Fig. 3). Apart from shared factors required for differentiation toward both the urothelium and urethral epithelium, urethral development requires the presence of androgens and activators of the TGF $\beta$ signaling pathway. The latter pathway should be activated with caution since TGF $\beta$ can activate the epithelial-tomesenchymal transition and thereby inducing fibroblast-like phenotype instead of epithelial phenotype.

The relevance of retinoic acid and PPAR $\gamma$ activation in directed differentiation toward urethral epithelium should be experimentally explored and, as urothelium and urethral epithelium share a common ancestor in the UGS, the process of transdifferentiation ${ }^{65}$ from urothelium to urethral epithelium is worth exploring. Differentiation could be tracked by markers described in this review (Fig. 3B), but we suggest to further develop an expression signature specific for urethral epithelium as described above.

Tissue morphology not only depends on the biological and chemical environment but also on physical factors. This might explain why, while most biological signaling pathways are shared, the morphology of urothelium (transitional epithelium) differs from urethral epithelium (pseudostratified). The bladder has a storage function and the surface area is varying with the amount of urine stored, while the surface area of the urethra is more constant. Transitional epithelium has the capacity to change its surface area more 
than pseudostratified epithelium. To determine the role of physical and mechanical factors on the epithelial morphology, it would be interesting to see if TE constructs seeded with urothelium change into a different epithelial morphology in vivo over time after grafting in the urethra, as observed in oral mucosa grafts in a rat model. ${ }^{72}$

The present review is limited by the availability of the published studies. All selected studies (apart from only one study $^{6}$ ) were either focusing on urothelium or on urethral epithelium, but not comparing both. In addition, a large variety of techniques were used on diverse species; fundamental early cellular processes are likely universal, however, ultimately, penile anatomy is very different between rodents and man, so signaling from the different tissue compartments with regard to development of the human urethra could be different.

\section{Conclusions}

Urothelium and urethral epithelium both derive from the endoderm and from the UGS. Therefore, in their development and differentiation, they both depend on signaling pathways such as Shh, BMP, Wnt, and FGF. While urothelium is a transitional epithelium and urethral epithelium is pseudostratified, they share p63 expression and several cytokeratins. Expression of uroplakins and keratin20 is unique for urothelium. To distinguish the urothelium from urethral epithelium, an expression signature should be developed.

Knowledge of the developmental cues leading to mature bladder urothelium and urethral epithelium may enhance engineering mature urothelium and urethral epithelium in vitro (Fig. 3). ${ }^{69-71}$ The methodology of directed (trans) differentiation may be used to allow epithelial cells to differentiate in the desired direction.

In conclusion, comparing urethra and bladder development with specific emphasis on the similarities and differences of urethral epithelium versus urothelium development provides insight on which future protocols for in vitro generation of urethral epithelium can be based.

\section{Acknowledgment}

This collaborative work was supported by the EU Regenerative Sphincter Therapy (ReST) project (COST Action BM1209).

\section{Disclosure Statement}

No competing financial interests exist.

\section{References}

1. Palmer, B.W., and Kropp, B.P. Update on tissue engineering in pediatric urology. Curr Urol Rep 14, 327, 2013.

2. Staack, A., Hayward, S.W., Baskin, L.S., and Cunha, G.R. Molecular, cellular and developmental biology of urothelium as a basis of bladder regeneration. Differentiation 73, 121, 2005.

3. de Kemp, V., de Graaf, P., Fledderus, J.O., Ruud Bosch, J.L., and de Kort, LM. Tissue engineering for human urethral reconstruction: systematic review of recent literature. PloS One 10, e0118653, 2015.

4. Lenas, P., Moos, M., and Luyten, F.P. Developmental engineering: a new paradigm for the design and manufacturing of cell-based products. Part I: from three-dimensional cell growth to biomimetics of in vivo development. Tissue Eng Part B Rev 15, 381, 2009.

5. Moher, D., Liberati, A., Tetzlaff, J., and Altman, D.G. Preferred reporting items for systematic reviews and metaanalyses: the PRISMA statement. PLoS Med 6, e1000097, 2009.

6. Bitgood, M.J., and McMahon, A.P. Hedgehog and Bmp genes are coexpressed at many diverse sites of cell-cell interaction in the mouse embryo. Dev Biol 172, 126, 1995.

7. Tanaka, S.T., Ishii, K., Demarco, R.T., Pope, J.C., Brock, J.W., 3rd, and Hayward, S.W. Endodermal origin of bladder trigone inferred from mesenchymal-epithelial interaction. J Urol 183, 386, 2010.

8. Carpenter, A., Paulus, A., Robinson, M., et al. 3 Dimensional morphometric analysis of murine bladder development and dysmorphogenesis. Dev Dyn 241, 522, 2012.

9. De la Rosette, J., Smedts, F., Schoots, C., Hoek, H., and Laguna, P. Changing patterns of keratin expression could be associated with functional maturation of the developing human bladder. J Urol 168, 709, 2002.

10. Erman, A., Veranic, P., Psenicnik, M., and Jezernik, K. Superficial cell differentiation during embryonic and postnatal development of mouse urothelium. Tissue Cell 38, 293, 2006.

11. Rudat, C., Grieskamp, T., Rohr, C., et al. Upk3b is dispensable for development and integrity of urothelium and mesothelium. PloS One 9, e112112, 2014.

12. Colopy, S.A., Bjorling, D.E., Mulligan, W.A., and Bushman, W. A population of progenitor cells in the basal and intermediate layers of the murine bladder urothelium contributes to urothelial development and regeneration. Dev Dyn 243, 988, 2014.

13. Gandhi, D., Molotkov, A., Batourina, E., et al. Retinoid signaling in progenitors controls specification and regeneration of the urothelium. Dev Cell 26, 469, 2013.

14. Sun, W., Wilhelmina Aalders, T., and Oosterwijk, E. Identification of potential bladder progenitor cells in the trigone. Dev Biol 393, 84, 2014.

15. Pignon, J.C., Grisanzio, C., Geng, Y., Song, J., Shivdasani, R.A., and Signoretti, S. P63-expressing cells are the stem cells of developing prostate, bladder, and colorectal epithelia. Proc Natl Acad Sci U S A 110, 8105, 2013.

16. Karni-Schmidt, O., Castillo-Martin, M., HuaiShen, T., et al. Distinct expression profiles of p63 variants during urothelial development and bladder cancer progression. Am J Pathol 178, 1350, 2011.

17. Cheng, W., Jacobs, W.B., Zhang, J.J.R., et al. $\Delta$ Np63 plays an anti-apoptotic role in ventral bladder development. Development 133, 4783, 2006.

18. Wilkins, S., Zhang, K.W., Mahfuz, I., et al. Insertion/ deletion polymorphisms in the DeltaNp63 promoter are a risk factor for bladder exstrophy epispadias complex. PLoS Genet 8, e1003070, 2012.

19. Baskin, L.S., Hayward, S.W., Young, P., and Cunha, G.R. Role of mesenchymal-epithelial interactions in normal bladder development. J Urol 156, 1820, 1996.

20. Baskin, L., DiSandro, M., Li, Y., Li, W., Hayward, S., and Cunha, G. Mesenchymal-epithelial interactions in bladder smooth muscle development: effects of the local tissue environment. J Urol 165, 1283, 2001.

21. DiSandro, M.J., Li, Y., Baskin, L.S., Hayward, S., and Cunha, G. Mesenchymal-epithelial interactions in bladder smooth muscle development: epithelial specificity. J Urol 160(3 II), 1040, 1998. 
22. Shiroyanagi, Y., Liu, B., Cao, M., et al. Urothelial sonic hedgehog signaling plays an important role in bladder smooth muscle formation. Differentiation 75, 968, 2007.

23. Jenkins, D., Winyard, P.J., and Woolf, A.S. Immunohistochemical analysis of Sonic hedgehog signalling in normal human urinary tract development. J Anatomy 211, 620, 2007.

24. Cao, M., Liu, B., Cunha, G., and Baskin, L. Urothelium patterns bladder smooth muscle location. Pediatr Res 64, 352, 2008.

25. Cao, M., Tasian, G., Wang, M.H., Liu, B., Cunha, G., and Baskin, L. Urothelium-derived Sonic hedgehog promotes mesenchymal proliferation and induces bladder smooth muscle differentiation. Differentiation 79, 244, 2010.

26. Burgu, B., McCarthy, L.S., Shah, V., Long, D.A., Wilcox, D.T., and Woolf, A.S. Vascular endothelial growth factor stimulates embryonic urinary bladder development in organ culture. BJU Int 98, 217, 2006.

27. Burgu, B., Medina Ortiz, W.E., Pitera, J.E., Woolf, A.S., and Wilcox, D.T. Vascular endothelial growth factor mediates hypoxic stimulated embryonic bladder growth in organ culture. J Urol 177, 1552, 2007.

28. DiSandro, M.J., Baskin, L.S., Li, Y.W., Werb, Z., and Cunha, G.R. Development and regenerative ability of bladder in the transgenic epidermal growth factor receptor gene knockout mouse. J Urol 158(3 Suppl), 1058, 1997.

29. Vinter-Jensen, L., and Nielsen, K. The effects of exogenous epidermal growth factor on the developing urinary tract in rats: a stereological description. Urol Res 26, 105, 1998.

30. Vinter-Jensen, L., and Orntoft, T.F. Glycoproteins in the urothelium and in the urine of the epidermal growth factor induced growing urinary tract in rats. Urol Res 26, 97, 1998.

31. Abler, L.L., Keil, K.P., Mehta, V., Joshi, P.S., Schmitz, C.T., and Vezina, C.M. A high-resolution molecular atlas of the fetal mouse lower urogenital tract. Dev Dyn 240, 2364, 2011.

32. Georgas, K.M., Armstrong, J., Keast, J.R., et al. An illustrated anatomical ontology of the developing mouse lower urogenital tract. Development (Cambridge) 142, 1893, 2015.

33. Thomas, J.C., Oottamasathien, S., Makari, J.H., et al. Temporal-spatial protein expression in bladder tissue derived from embryonic stem cells. J Urol 180(4 Suppl), 1784, 2008.

34. Chung, P.J., Chi, L.M., Chen, C.L., et al. MicroRNA-205 targets tight junction-related proteins during urothelial cellular differentiation. MCP 13, 2321, 2014.

35. Kurzrock, E.A., Baskin, L.S., and Cunha, G.R. Ontogeny of the male urethra: theory of endodermal differentiation. Differentiation 64, 115, 1999.

36. Hynes, P.J., and Fraher, J.P. The development of the male genitourinary system: II. The origin and formation of the urethral plate. Br J Plast Surg 57, 112, 2004.

37. Chiu, H.S., Szucsik, J.C., Georgas, K.M., et al. Comparative gene expression analysis of genital tubercle development reveals a putative appendicular Wnt7 network for the epidermal differentiation. Dev Biol 344, 1071, 2010.

38. Petiot, A., Perriton, C.L., Dickson, C., and Cohn, M.J. Development of the mammalian urethra is controlled by Fgfr2-IIIb. Development 132, 2441, 2005.

39. Hynes, P.J., and Fraher, J.P. The development of the male genitourinary system: III. The formation of the spongiose and glandar urethra. Br J Plast Surg 57, 203, 2004.

40. Hadidi, A.T., Roessler, J., and Coerdt, W. Development of the human male urethra: a histochemical study on human embryos. J Pediatr Surg 49, 1146, 2014.

41. Li, Y., Sinclair, A., Cao, M., et al. Canalization of the urethral plate precedes fusion of the urethral folds during male penile urethral development: the double zipper hypothesis. J Urol 193, 1353, 2015.

42. Ogino, Y., Suzuki, K., Haraguchi, R., Satoh, Y., Dolle, P., and Yamada, G. External genitalia formation: role of fibroblast growth factor, retinoic acid signaling, and distal urethral epithelium. Ann N Y Acad Sci 948, 13, 2001.

43. Seifert, A.W., Harfe, B.D., and Cohn, M.J. Cell lineage analysis demonstrates an endodermal origin of the distal urethra and perineum. Dev Biol 318, 143, 2008.

44. Seifert, A.W., Bouldin, C.M., Choi, K.S., Harfe, B.D., and Cohn, M.J. Multiphasic and tissue-specific roles of sonic hedgehog in cloacal septation and external genitalia development. Development 136, 3949, 2009.

45. Perriton, C.L., Powles, N., Chiang, C., Maconochie, M.K., and Cohn, M.J. Sonic hedgehog signaling from the urethral epithelium controls external genital development. Dev Biol 247, 26, 2002.

46. Wu, X., Ferrara, C., Shapiro, E., and Grishina, I. Bmp7 expression and null phenotype in the urogenital system suggest a role in re-organization of the urethral epithelium. GEP 9, 224, 2009.

47. Morgan, E.A., Nguyen, S.B., Scott, V., and Stadler, H.S. Loss of Bmp7 and Fgf8 signaling in Hoxa13-mutant mice causes hypospadia. Development 130, 3095, 2003.

48. Miyagawa, S., Matsumaru, D., Murashima, A., et al. The role of sonic hedgehog-Gli2 pathway in the masculinization of external genitalia. Endocrinology 152, 2894, 2011.

49. Sajjad, Y., Quenby, S., Nickson, P., Lewis-Jones, D.I., and Vince, G. Immunohistochemical localization of androgen receptors in the urogenital tracts of human embryos. Reproduction 128, 331, 2004.

50. Chen, H., Yong, W., Hinds, T.D., Jr., et al. Fkbp52 regulates androgen receptor transactivation activity and male urethra morphogenesis. J Biol Chem 285, 27776, 2010.

51. Kim, K.S., Liu, W., Cunha, G.R., et al. Expression of the androgen receptor and 5 alpha-reductase type 2 in the developing human fetal penis and urethra. Cell Tissue Res 307, 145, 2002.

52. Jesmin, S., Mowa, C.N., Matsuda, N., et al. Evidence for a potential role of estrogen in the penis: detection of estrogen receptor-alpha and -beta messenger ribonucleic acid and protein. Endocrinology 143, 4764, 2002.

53. Satoh, Y., Haraguchi, R., Wright, T.J., et al. Regulation of external genitalia development by concerted actions of FGF ligands and FGF receptors. Anat Embryol 208, 479, 2004.

54. Haraguchi, R., Suzuki, K., Murakami, R., et al. Molecular analysis of external genitalia formation: the role of fibroblast growth factor (Fgf) genes during genital tubercle formation. Development 127, 2471, 2000.

55. Ching, S.T., Cunha, G.R., Baskin, L.S., Basson, M.A., and Klein, O.D. Coordinated activity of Spry1 and Spry2 is required for normal development of the external genitalia. Dev Biol 386, 1, 2014.

56. Seifert, A.W., Yamaguchi, T., and Cohn, M.J. Functional and phylogenetic analysis shows that Fgf8 is a marker of genital induction in mammals but is not required for external genital development. Development 136, 2643, 2009.

57. Beleza-Meireles, A., Lundberg, F., Lagerstedt, K., et al. FGFR2, FGF8, FGF10 and BMP7 as candidate genes for hypospadias. Eur J Hum Genet 15, 405, 2007.

58. Gredler, M.L., Seifert, A.W., and Cohn, M.J. Tissuespecific roles of Fgfr2 in development of the external genitalia. Development 142, 2203, 2015. 
59. Harada, M., Omori, A., Nakahara, C., Nakagata, N., Akita, K., and Yamada, G. Tissue-specific roles of FGF signaling in external genitalia development. Dev Dyn 244, 759, 2015.

60. Li, J., Willingham, E., and Baskin, L.S. Gene expression profiles in mouse urethral development. BJU Int 98, 880, 2006.

61. Pechriggl, E.J., Bitsche, M., Blumer, M.J., and Fritsch, H. The male urethra: spatiotemporal distribution of molecular markers during early development. Ann Anat 195, 260, 2013.

62. Erol, A., Baskin, L.S., Li, Y.W., and Liu, W.H. Anatomical studies of the urethral plate: why preservation of the urethral plate is important in hypospadias repair. BJU Int $\mathbf{8 5}$, 728, 2000.

63. Armfield, B.A., Seifert, A.W., Zheng, Z., et al. Molecular characterization of the genital organizer: gene expression profile of the mouse urethral plate epithelium. J Urol 196, 1295, 2016.

64. Osborn, S.L., and Kurzrock, E.A. Production of urothelium from pluripotent stem cells for regenerative applications. Curr Urol Rep 16, 466, 2015.

65. Cohen, D.E., and Melton, D. Turning straw into gold: directing cell fate for regenerative medicine. Nat Rev Genet 12, 243, 2011.

66. D'Alessio, A.C., Fan, Z.P., Wert, K.J., et al. A systematic approach to identify candidate transcription factors that control cell identity. Stem Cell Rep 5, 763, 2015.

67. Cahan, P., Li, H., Morris, S.A., Lummertz da Rocha, E., Daley, G.Q., and Collins, J.J. CellNet: network biology applied to stem cell engineering. Cell 158, 903, 2014.

68. Rackham, O.J., Firas, J., Fang, H., et al. A predictive computational framework for direct reprogramming between human cell types. Nat Genet 48, 331, 2016.
69. Kang, M., Kim, H.H., and Han, Y.M. Generation of bladder urothelium from human pluripotent stem cells under chemically defined serum- and feeder-free system. Int $\mathbf{J}$ Mol Sci 15, 7139, 2014.

70. Osborn, S.L., Thangappan, R., Luria, A., Lee, J.H., Nolta, J., and Kurzrock, E.A. Induction of human embryonic and induced pluripotent stem cells into urothelium. Stem Cells Transl Med 3, 610, 2014.

71. Wezel, F.D.A., Novak, D., Larribere, L., Galach, M., Unger, C., and Utikal, J. Directed differentiation of human induced pluripotent stem cells (hiPS) for urothelial cellbased tissue-engineering therapies. Eur Urol Supplementals 14, e1006, 2015.

72. Martin-Cano, F., Garzon, I., Maranes, C., et al. Histological and immunohistochemical changes in the rat oral mucosa used as an autologous urethral graft. J Pediatr Surg 48, 1557, 2013.

Address correspondence to:

Petra de Graaf, PhD

Department of Urology

University Medical Centre Utrecht

HP. C04.236, PO Box 85500

Utrecht 3508 GA

The Netherlands

E-mail: p.degraaf-4@umcutrecht.nl

Received: August 30, 2016

Accepted: October 28, 2016

Online Publication Date: December 2, 2016 